\title{
Split Algorithms for New Implicit Feasibility Null-Point Problems
}

\author{
Abdellatif Moudafi ${ }^{1}$ and Muhammad Aslam Noor ${ }^{2, *}$ \\ ${ }^{1}$ Université des Antilles et de Guyane, Ceregmia-DSI 97200 Schoelcher, Martinique, France \\ ${ }^{2}$ Mathematics Department, COMSATS Institute of Information Technology, Park Road, Islamabad, Pakistan
}

Received: 7 Aug. 2013, Revised: 4 Nov. 2013, Accepted: 5 Nov. 2013

Published online: 1 Sep. 2014

\begin{abstract}
Inspired by the very recent work by Noor and Noor [9] and given a closed convex set-valued mapping $C$, we propose a split algorithm for solving the problem of finding an element $x^{*}$ which is a zero of a given maximal monotone operator $T$ such that its image, $A x^{*}$, under a linear operator, $A$, is in a closed convex set $C\left(x^{*}\right)$. Then, we present two strong convergence results and state some examples as applications. The ideas and techniques of this paper may motivate the readers to discover some novel and innovative applications of the implicit split feasibility problems in various branches of pure and applied sciences.
\end{abstract}

Keywords: Fixed-point, monotone operator, implicit feasibility problem.

2010 AMS Subject Classification: Primary, 49J53, 65K10; Secondary, 49M37, 90C25.

\section{Introduction and preliminaries}

Throughout, $H$ is a Hilbert space, $\langle\cdot, \cdot\rangle$ denotes the inner product and $\|\cdot\|$ stands for the corresponding norm. The split feasibility problem (SFP) has received much attention due to its applications in image denoising, signal processing and image reconstruction, with particular progress in intensity-modulated therapy. For a complete and exhaustive study on algorithms for solving convex feasibility problem, including comments about their applications and an excellent bibliography see, for example [1] and for split convex feasibility problem see, for instance, the excellent paper [5] and the references therein. Inspired by the idea developed in [9], our interest in this paper is on the study of the convergence of an algorithm for solving a Implicit Feasibility Null-point Problem, i.e., the case where the constrained set, instead of being fixed, is a set-valued mapping. Besides being a more general case, it also has many applications, see for example [2]). Note that by taking $A=I$, the identity mapping and $T=\partial \phi$, the subdifferential of a strongly convex proper lower semi-continuous function $\phi$, problem (4) reduces to finding a common element in $\operatorname{argmin} \phi$ and the implicit convex set $C(x)$. To be in a position to apply the fixed-point Banach principle and by observing that the fixed-point reformulation of the problem considered in [9] involves the projection operator over convex sets and that the techniques are strongly based on its properties which do not depend on any parameter in contrast to the resolvent and proximal mappings. In this paper, we introduce and consider a new implicit feasibility null-point problems. We suggest and analyze some split algorithms for solving this new feasibility problem. Strong convergence of the proposed algorithm is discussed under some suitable conditions. Some applications of this new problem are given. Comparison of the proposed methods with other techniques is an interesting problem for future research.

To begin with, let us recall that the split feasibility problem (SFP) is to find a point

$x \in C$ such that $A x \in Q$,

where $C$ is a closed convex subset of a Hilbert space $H_{1}$, $Q$ is a closed convex subset of a Hilbert space $H_{2}$, and $A: H_{1} \rightarrow H_{2}$ is a bounded linear operator.

Assuming that the (SFP) has a solution, it is no hard to see that $x \in C$ solves (1) if and only if it solves to fixed-point equation

$x=P_{C}\left(I-\gamma A^{*}\left(I-P_{Q}\right) A\right) x, x \in C$,

where $P_{C}$ and $P_{Q}$ are the (orthogonal) projection onto $C$ and $Q$, respectively, $\gamma>0$ is any positive constant and $A^{*}$

\footnotetext{
*Corresponding author e-mail: noormaslam@hotmail.com
} 
denotes the adjoint of $A$.

To solve the (2), Byrne [4] proposed the CQ algorithm which generates a sequence $\left(x_{k}\right)$ by

$x_{k+1}=P_{C}\left(I-\gamma A^{*}\left(I-P_{Q}\right) A\right) x_{k}, k \in \mathbb{N}$,

where $\gamma \in(0,2 / \lambda)$ with $\lambda$ being the spectral radius of the operator $A^{*} A$.

\section{Main Results}

In the sequel, we will focus our attention on the following implicit feasibility null-point problem

find $x^{*} \in T^{-1}(0)$ such that $A x^{*} \in C\left(x^{*}\right)$,

where $A: H_{1} \rightarrow H_{2}$ is a bounded linear operator, $T: H_{1} \rightarrow$ $H_{1}$ a maximal monotone operator and $C: H_{1} \rightarrow 2^{H_{2}}$ is a set-valed map with closed convex values.

Fact 1. If $T$ is strongly monotone with constant $\alpha$, then (see for example [10])

$$
\left\|J_{\lambda}^{T}(x)-J_{\lambda}^{T}(y)\right\| \leq \frac{1}{1+\alpha \lambda}\|x-y\|, \quad \forall x, y .
$$

Fact 2. It is well-known, see for example [11], that if $C$ is a closed convex set, then $A^{*}\left(I-P_{C}\right) A$ is an inverse strongly monotone operator, namely

$$
\begin{aligned}
& \left\langle A^{*}\left(I-P_{C}\right) A x-A^{*}\left(I-P_{C}\right) A y, x-y\right\rangle \\
& \geq \frac{1}{\|A\|^{2}}\left\|A^{*}\left(I-P_{C}\right) A x-A^{*}\left(I-P_{C}\right) A y\right\|
\end{aligned}
$$

and thus $A^{*}\left(I-P_{C}\right) A$ is Lipschitz with constant $\|A\|^{2}$.

Fact 3. It is easy to see that if $x^{*}$ solves (4), then $x^{*}$ verifies the following fixed-point problem

$$
x^{*}=J_{\lambda_{k}}^{T}\left(x^{*}-\gamma A^{*}\left(I-P_{C\left(x^{*}\right)}\right) A x^{*}\right) .
$$

Fact 4. $x^{*}$ solves (4) if, and only if, $x^{*}$ verifies the following fixed-point formulation

$$
x^{*}=J_{\lambda_{k}}^{T}\left(x^{*}-\gamma A^{*}\left(I-P_{C\left(x^{*}\right)}\right) A x^{*}\right) .
$$

It is easy to see that if $x^{*}$ solves (4), then it also satisfies the fixed-point problem. Conversely, assume that $x^{*}$ verifies the fixed-point problem. We then get the following, by using the definition of the resolvent operator

$$
\begin{gathered}
x^{*}-\gamma A^{*}\left(I-P_{C\left(x^{*}\right)}\right) A x^{*} \in x^{*}+\lambda_{k} T x^{*} \\
\left.\Leftrightarrow-\gamma / \lambda_{k} A^{*}\left(I-P_{C\left(x^{*}\right)}\right)\right) A x^{*} \in T x^{*} .
\end{gathered}
$$

By virtue of the monotonicity of $T$, we can write

$\left\langle y+\gamma / \lambda_{k} A^{*}\left(I-P_{C\left(x^{*}\right)}\right) A x^{*}, x^{*}-x\right\rangle \leq 0 \forall x \in D(T), \forall y \in T x$.

On the other hand, by Fact 3, we have

$$
\gamma / \lambda_{k}\left\langle A x^{*}-P_{C\left(x^{*}\right)} A x^{*}, v-P_{C\left(x^{*}\right)} A x^{*}\right\rangle \leq 0 \forall v \in C\left(x^{*}\right) .
$$

Adding up the last two inequalities, we get

$$
\begin{aligned}
& \left\langle y, x^{*}-x\right\rangle \\
& +\gamma / \lambda_{k}\left\langle A x^{*}-P_{C\left(x^{*}\right)} A x^{*}, A x^{*}-P_{C\left(x^{*}\right)} A x^{*}+v-A x\right\rangle \leq 0 \\
& \forall v \in C\left(x^{*}\right), \forall x \in D(T) .
\end{aligned}
$$

It turns out that

$$
\begin{aligned}
& \left\|A x^{*}-P_{C\left(x^{*}\right)} A x^{*}\right\|^{2} \\
& \leq\left\langle A x^{*}-P_{C\left(x^{*}\right)} A x^{*}, A x-v\right\rangle+\lambda_{k} / \gamma\left\langle y, x-x^{*}\right\rangle .
\end{aligned}
$$

Thus substituting $x^{*}$ for $x$ and $A x^{*}$ for $v$ in the last inequality, we obtain

$\left\|A x^{*}-P_{C\left(x^{*}\right)} A x^{*}\right\|^{2}=0$, hence $A x^{*}=P_{C\left(x^{*}\right)} A x^{*} \in C\left(x^{*}\right)$.

The fixed-point formulation reduces to $x^{*}=J_{\lambda_{k}}^{T}\left(x^{*}\right)$, thus $x^{*} \in T^{-1}(0)$.

To solve (4), we suggest the use of the following algorithm:

Algorithm (IFNA): Initialization: Let $\lambda_{0}>0$ and $x_{0} \in H_{1}$ be arbitrary.

Iterative step:

$x_{k+1}=J_{\lambda_{k}}^{T}\left(x_{k}-\gamma A^{*}\left(I-P_{C\left(x_{k}\right)}\right) A x_{k}\right), k \in \mathbb{N}$,

where $v_{k}$ and $\beta$ will be defined in the sequel. result

We are now in a position to prove our convergence

Theorem 1. Given a bounded linear operator A : $H_{1} \rightarrow H_{2}, H_{1}, H_{2}$ two Hilbert spaces, $T: H_{2} \rightarrow 2^{H_{2}}$ is a $\alpha$-strongly monotone set-valued operator and $C: H_{1} \rightarrow 2^{H_{2}}$ is a set-valued mapping with closed convex values. If

$\left\|P_{C(x)} z-P_{C(y)} z\right\| \leq \beta\|x-y\|, \forall x, y, z$,

where $\beta>0$ is a constant, then any sequence $\left(x_{k}\right)$ generated by the algorithm (5) strongly converges to the unique solution of (4) provided that

$\beta \in] 0,1\left[\right.$ and $0<\lambda_{k}<\frac{1}{\gamma \alpha\|A\|(\|A\|+\beta)}$.

Proof. Set $v_{k}:=\frac{\alpha \lambda_{k}}{1+\alpha \lambda_{k}}$ and let $x^{*}$ be the solution to (4), then by Fact 3 , we have $x^{*}=J_{\lambda_{k}}^{T}\left(x^{*}-\gamma A^{*}\left(I-P_{C\left(x^{*}\right)}\right) A x^{*}\right)$. By Facts 1 and 2, we know that $J_{\lambda_{k}}^{T}$ is a contraction with constant $v_{k}$ and $A^{*}\left(I-P_{C}\right) A$ is Lipschitz continuous with constant $\|A\|^{2}$. Therefore, we successively have

$$
\begin{aligned}
& \left\|x_{k+1}-x^{*}\right\| \\
= & \| J_{\lambda_{k}}^{T}\left(x_{k}-\gamma A^{*}\left(I-P_{C\left(x_{k}\right)}\right) A x_{k}\right) \\
& -J_{\lambda_{k}}^{T}\left(x^{*}-\gamma A^{*}\left(I-P_{C\left(x^{*}\right)}\right) A x^{*}\right) \| \\
\leq & v_{k}\left\|x_{k}-x^{*}-\gamma\left(A^{*}\left(I-P_{C\left(x_{k}\right)}\right) A x_{k}-A^{*}\left(I-P_{C\left(x^{*}\right)}\right) A x^{*}\right)\right\| \\
\leq & v_{k}\left(\left\|x_{k}-x^{*}\right\|\right.
\end{aligned}
$$




$$
\begin{aligned}
& +\gamma\left(\left\|A^{*}\left(I-P_{C\left(x_{k}\right)}\right) A x_{k}-A^{*}\left(I-P_{C\left(x_{k}\right)}\right) A x^{*}\right\|\right. \\
+ & \left.\left.\left.\| A^{*}\left(I-P_{C\left(x_{k}\right)}\right) A x^{*}-A^{*}\left(I-P_{C\left(x^{*}\right)}\right) A x^{*}\right)\right) \|\right) \\
\leq & v_{k}\left(\left\|x_{k}-x^{*}\right\|+\gamma\left(\|A\|^{2}\left\|x_{k}-x^{*}\right\|+\|A\| \beta\left\|x_{k}-x^{*}\right\|\right)\right) \\
\leq & v_{k}(1+\gamma(\|A\|+\beta))\|A\|\left\|x_{k}-x^{*}\right\|,
\end{aligned}
$$

$\lambda_{k}$ was chosen judiciously such that $\theta:=v_{k}(1+\gamma(\|A\|+$ $\beta)) \in] 0,1[$. The latter assures the strong convergence of $\left(x_{k}\right)$ to $x^{*}$ the unique solution of (2.4).

Now, we propose a second convergence result.

Theorem 2. Given a bounded linear operator A $: H_{1} \rightarrow H_{2}, H_{1}, H_{2}$ two Hilbert spaces, $T: H_{2} \rightarrow 2^{H_{2}}$ is a $\alpha$-strongly monotone set-valued operator and $C: H_{1} \rightarrow 2^{H_{2}}$ is a set-valued mapping with closed convex values. If

$$
\left\|P_{C(x)} z-P_{C(y)} z\right\| \leq \beta\|x-y\|, \forall x, y, z
$$

then any sequence $\left(x_{k}\right)$ generated by the algorithm (5) strongly converges to the unique solution of (4), provided that

$\beta \in] 0,1\left[, 0<\lambda_{k}<\frac{1}{\alpha(-1+\sqrt{1+2 \gamma \beta\|A\|})}\right.$

and

$$
0<\gamma<2 /\|A\|^{2}
$$

Proof. Set $v_{k}:=\frac{\alpha \lambda_{k}}{1+\alpha \lambda_{k}}$ and set $x^{*}$ be the solution to (4), then $J_{\lambda_{k}}^{T}\left(x^{*}-\gamma A^{*}\left(I-P_{C\left(x^{*}\right)}\right) A x^{*}=x^{*}\right.$. By Facts 1 and 2, we know that $J_{\lambda_{k}}^{T}$ is a contraction with constant $v_{k}$ and $A^{*}(I-$ $\left.P_{C}\right) A$ is inverse strongly monotone with constant $1 /\|A\|^{2}$. Therefore, we successively have

$$
\begin{aligned}
& \left\|x_{k+1}-x^{*}\right\|^{2} \\
= & \| J_{\lambda_{k}}^{T}\left(x_{k}-\gamma A^{*}\left(I-P_{C\left(x_{k}\right)}\right) A x_{k}\right) \\
& -J_{\lambda_{k}}^{T}\left(x^{*}-\gamma A^{*}\left(I-P_{C\left(x^{*}\right)}\right) A x^{*}\right) \|^{2} \\
\leq & v_{k}^{2}\left\|x_{k}-x^{*}-\gamma\left(A^{*}\left(I-P_{C\left(x_{k}\right)}\right) A x_{k}-A^{*}\left(I-P_{C\left(x^{*}\right)}\right) A x^{*}\right)\right\|^{2} \\
= & v_{k}^{2}\left(\left\|x_{k}-x^{*}\right\|^{2}\right. \\
& -2 \gamma\left\langle A^{*}\left(I-P_{C\left(x_{k}\right)}\right) A x_{k}-A^{*}\left(I-P_{C\left(x^{*}\right)}\right) A x^{*}, x_{k}-x^{*}\right\rangle \\
+ & \left.\gamma^{2}\left\|A^{*}\left(I-P_{C\left(x_{k}\right)}\right) A x_{k}-A^{*}\left(I-P_{C\left(x^{*}\right)}\right) A x^{*}\right\|^{2}\right) \\
= & v_{k}^{2}\left(\left\|x_{k}-x^{*}\right\|^{2}-2 \gamma\left\langle A^{*}\left(I-P_{C\left(x_{k}\right)}\right) A x_{k}\right.\right. \\
& \left.-A^{*}\left(I-P_{C\left(x_{k}\right)}\right) A x^{*}, x_{k}-x^{*}\right\rangle \\
& -2 \gamma\left\langle A^{*}\left(I-P_{C\left(x_{k}\right)}\right) A x^{*}-A^{*}\left(I-P_{C\left(x^{*}\right)}\right) A x^{*}, x_{k}-x^{*}\right\rangle \\
& \left.+\gamma^{2}\left\|A^{*}\left(I-P_{C\left(x_{k}\right)}\right) A x_{k}-A^{*}\left(I-P_{C\left(x^{*}\right)}\right) A x^{*}\right\|^{2}\right) \\
\leq & v_{k}^{2}\left(\left\|x_{k}-x^{*}\right\|^{2}-2 \gamma /\|A\|^{2} \| A^{*}\left(I-P_{C\left(x_{k}\right)}\right) A x_{k}\right. \\
& -A^{*}\left(I-P_{C\left(x^{*}\right)}\right) A x^{*} \|^{2} \\
& +2 \gamma\|A\| \beta\left\|x_{k}-x^{*}\right\|^{2}+\gamma^{2} \| A^{*}\left(I-P_{C\left(x_{k}\right)}\right) A x_{k} \\
& \left.-A^{*}\left(I-P_{C\left(x^{*}\right)}\right) A x^{*} \|^{2}\right)
\end{aligned}
$$

$$
\begin{array}{r}
\leq v_{k}^{2}(1+2 \gamma\|A\| \beta)\left\|x_{k}-x^{*}\right\|^{2} \\
-\gamma\left(2 /\|A\|^{2}-\gamma\right) v_{k}^{2} \| A^{*}\left(I-P_{C\left(x_{k}\right)}\right) A x_{k} \\
-A^{*}\left(I-P_{C\left(x^{*}\right)}\right) A x^{*} \|^{2},
\end{array}
$$

$\lambda_{k}$ was chosen judiciously such that $\left.\theta:=v_{k}^{2}(1+2 \gamma \beta\|A\|) \in\right] 0,1[$. The latter assures the strong convergence of $\left(x_{k}\right)$ to $x^{*}$ the unique solution of (4). Moreover, we have

$$
\lim _{k \rightarrow+\infty} v_{k}\left\|A^{*}\left(I-P_{C\left(x_{k}\right)}\right) A x_{k}-A^{*}\left(I-P_{C\left(x^{*}\right)}\right) A x^{*}\right\|=0 .
$$

From which we infer, if in addition we assume that $\inf _{k} \lambda_{k}>0$, that the sequence $\left(A^{*}\left(I-P_{C\left(x_{k}\right)}\right) A x_{k}\right)$ strongly converges to $A^{*}\left(I-P_{C\left(x^{*}\right)}\right) A x^{*}$.

It is worth mentioning that we can develop the same analysis for the following implicit feasibility fixed-point problem

find $x^{*} \in F i x P$ such that $A x^{*} \in C\left(x^{*}\right)$,

where $\mathrm{P}: \mathrm{H}_{2} \rightarrow \mathrm{H}_{2}$ is a $\kappa$-contraction, by considering the following algorithm

\section{Algorithm:}

Initialization: Let $x_{0} \in H_{1}$ be arbitrary.

Iterative step:

$x_{k+1}=P\left(x_{k}-\gamma A^{*}\left(I-P_{C\left(x_{k}\right)}\right) A x_{k}\right), k \in \mathbb{N}$.

Following the same lines of the proof of the above Theorem, we obtain

Proposition 1. Given a bounded linear operator A : $H_{1} \rightarrow H_{2}, H_{1}, H_{2}$ are two Hilbert spaces, $P: H_{2} \rightarrow 2^{H_{2}}$ a $\kappa$-contraction and $C: H_{1} \rightarrow 2^{H_{2}}$ a set-valued mapping with closed convex values. If

$$
\left\|P_{C(x)} z-P_{C(y)} z\right\| \leq \beta\|x-y\|, \forall x, y, z
$$

then any sequence $\left(x_{k}\right)$ generated by the algorithm (10) strongly converges to the unique solution of (9), provided that

$$
0<\gamma<\frac{(1-\kappa)}{\kappa\|A\|(\|A\|+\beta)}
$$

Following the same lines of the proof of the above Theorem, we obtain

Proposition 2. Given a bounded linear operator A : $H_{1} \rightarrow H_{2}, H_{1}, H_{2}$ are two Hilbert spaces, $P: H_{2} \rightarrow 2^{H_{2}}$ a $\kappa$-contraction and $C: H_{1} \rightarrow 2^{H_{2}}$ a set-valued mapping with closed convex values. If

$$
\left\|P_{C(x)} z-P_{C(y)} z\right\| \leq \beta\|x-y\|, \forall x, y, z
$$

then any sequence $\left(x_{k}\right)$ generated by the algorithm (10) strongly converges to the unique solution of (9), provided that $0<\gamma<\frac{\left(1-\kappa^{2}\right)}{\kappa^{2}\|A\| \beta}$. 
Remark. We would like to emphasize that our results can be extended to the case $T=T_{1}+T_{2}$, where $T_{1}$ is $\alpha$-strongly monotone and $T_{2}$ is $\theta$-Lipschitz continuous. Note that in the case the main operator $T$ is not necessarily monotone. In this setting, we introduce the following algorithm

$x_{k+1}=J_{\lambda_{k}}^{T_{1}}\left(x_{k}-\gamma A^{*}\left(I-P_{C\left(x_{k}\right)}\right) A x_{k}-\lambda_{k} T_{2} x_{k}\right), k \in \mathbb{N}$.

By replacing, for example in Theorem 2, the assumption $T$ strongly monotone by the hypotheses: $T_{1}$ is an $\alpha$-strongly monotone and $T_{2}$ is an $\theta$-Lipschitz continuous, and the condition on the parameters $\lambda_{k}$ by $0<\lambda_{k}<\frac{-\alpha \gamma\|A\|(\beta+\|A\|)+\sqrt{\alpha^{2} \gamma^{2}\|A\|^{2}(\beta+\|A\|)^{2}+4 \theta}}{2 \theta}$, we obtain, by adapting the proof of Theorem 1, the strong convergence of the sequence generated by (11) to the unique solution of (4). Indeed in this case we have

$$
\left\|x_{k+1}-x^{*}\right\| \leq v_{k}\left(\left(1+\gamma(\|A\|+\beta)\|A\|+\lambda_{k} \theta\right)\left\|x_{k}-x^{*}\right\| .\right.
$$

Remark. It is also worth mentioning, as the resolvent cannot be computed in general in a closed-form, that we can consider an approximate version of the initial algorithm to take into account errors computation, more precisely we can consider the following algorithm

$$
x_{k+1}=J_{\lambda_{k}}^{T}\left(x_{k}-\gamma A^{*}\left(I-P_{C\left(x_{k}\right)}\right) A x_{k}\right)+\varepsilon_{k}, k \in \mathbb{N} .
$$

It is easily seen, under the same assumption as in Theorem 2.1 with the condition on $\left(\lambda_{k}\right)$ replaced by $\lambda_{k} \geq \underline{\lambda}$, that $\left(x_{k}\right)$ still strongly converges to the solution of (2.4) as long as

$$
\lim _{k \rightarrow+\infty} \varepsilon_{k}=0 \text { and } 0<\gamma<\frac{\alpha \underline{\lambda}}{(\|A\|+\beta)\|A\|} .
$$

Indeed, in this case, we have

$$
\left\|x_{k+1}-x^{*}\right\| \leq \sigma_{k}\left\|x_{k}-x^{*}\right\|+\varepsilon_{k}
$$

with

$$
\begin{aligned}
\sigma_{k} & :=v_{k}(1+\gamma(\|A\|+\beta)\|A\|) \\
& \leq \sigma:=\frac{1}{1+\underline{\lambda} \alpha}(1+\gamma(\|A\|+\beta))\|A\| .
\end{aligned}
$$

It turns out that

$$
\left\|x_{k+1}-x^{*}\right\| \leq \sigma^{n+1}\left\|x_{0}-x^{*}\right\|+\sum_{j=1}^{n} \sigma^{n-j} \varepsilon_{j}
$$

and the result follows thanks to the well-known HardyCesaro Theorem on the convolution of real sequences.

\section{Applications}

Now, we consider the following special cases:

\section{Implicit feasibility minimization problem.}

Let $\phi: H_{1} \rightarrow \mathbb{R}$ be a lower semicontinuous convex function by setting $T=\partial \phi$ in (4), we obtain the following Quasi Split Minimization Problem (QSMP):

find $x^{*}=\operatorname{argmim} \phi$ such that $A x^{*} \in C\left(x^{*}\right)$

and (5) reduces to

$x_{k+1}=\operatorname{prox}_{\lambda_{k} \phi}\left(x_{k}-\gamma A^{*}\left(I-P_{C\left(x_{k}\right)}\right) A x_{k}\right), k \in \mathbb{N}$,

where $\operatorname{prox}_{\lambda_{k} \phi}(x):=\operatorname{argmin}_{y}\left\{\phi(y)+\frac{1}{2 \lambda}\|x-y\|^{2}\right\}$ is the proximal mapping of $\phi$. The assumption of strong monotonicity of $\partial \phi$ is equivalent to the strong convexity of $\phi$.

\section{Implicit feasibility Saddle-point problem.}

Let $X, Y$ be two Hilbert spaces, a function $L: X \times Y \rightarrow \mathbb{R} \cup\{-\infty,+\infty\}$ is convex-concave if it is convex in the variable $x$ and concave in the variable $y$. To such a function, Rockafellar associated the operator $T_{L}$, defined by

$$
T_{L}=\partial_{1} L \times \partial_{2}(-L),
$$

where $\partial_{1}$ (resp. $\partial_{2}$ ) stands for the subdifferential of $L$ with respect to the first (resp. the second) variable.

$T_{L}$ is a maximal monotone operator if and only if $L$ is closed and proper in Rockafellar sense (see, [10]). Moreover, it is well known that $\left(x^{*}, y^{*}\right)$ is a saddlepoint of $L$, namely

$$
L\left(x^{*}, y\right) \leq L\left(x^{*}, y^{*}\right) \leq L\left(x, y^{*}\right), \forall(x, y) \in X \times Y
$$

if and only if the following monotone variational inclusion holds true $(0,0) \in T_{L}\left(x^{*}, y^{*}\right)$.

Now, if in the (4) we set $H_{1}=X_{1} \times Y_{1}, H_{2}=X_{2} \times Y_{2}$, $T=T_{L}$ with $L$ be a proper closed convex-concave function, then we obtain the following Quasi Split Minimax Problem (QSMMP):

Find

$$
\begin{array}{r}
\left(x^{*}, y^{*}\right)=\operatorname{argminmax}_{(x, y) \in H_{1}} L(x, y) ; \\
A\left(x^{*}, y^{*}\right) \in C\left(x^{*}, y^{*}\right),
\end{array}
$$

and (5) reduces to

$$
\begin{aligned}
& \left(x_{k+1}, y_{k+1}\right)=\operatorname{prox}_{\lambda_{k} L}\left(x_{k}, y_{k}\right)\left(\left(x_{k}, y_{k}\right)\right. \\
& \left.\quad-\gamma A^{*}\left(I-P_{C\left(x_{k}, y_{k}\right.}\right) A\left(x_{k}, y_{k}\right)\right), k \in \mathbb{N},
\end{aligned}
$$

where

$$
\operatorname{prox}_{\lambda_{k} L}(x, y)
$$
$\left.\operatorname{argminmax}_{(u, v)}\{L(u, v))+\frac{1}{2 \lambda}\|x-u\|^{2}-\frac{1}{2 \lambda}\|y-v\|^{2}\right\}$. The assumption of strong monotonicity of $T_{L}$ is equivalent to the strong convexity of $L$ with respect to the first variable and its strong concavity with respect to the second one.

\section{Implicit feasibility equilibrium problem.}

Having in mind the connection between monotone 
operators and equilibrium functions, we may consider the following problem

$A_{F}(x) \ni 0$,

with $A_{F}$ defined as follows

$$
v \in A_{F}(x) \Leftrightarrow F(x, y)+\langle v, x-y\rangle \geq 0, \forall y \in D,
$$

$D$ is a closed convex set and $F: D \times D \rightarrow \mathbb{R}$ belongs in the class of bifunctions $F$ verifying the following usual conditions:

(A1) $F(x, x)=0$ for all $x, y \in D$;

(A2) $\quad F$ is monotone, i.e., $\quad F(x, y)+F(y, x) \leq$ 0 for all $x, y \in D$;

(A3) $\quad \limsup _{t \downarrow 0} F(t z+(1-t) x, y) \leq$ $F(x, y)$ for any $x, y, z \in D$;

(A4) for each $x \quad \in \quad D, y \quad \rightarrow$ $F(x, y)$ is convex and lower-semicontinuous.

It is well-known see [7] that $A_{F}$ is maximal monotone and that the associated resolvent operator $T_{\lambda}: H \rightarrow D$ is defined by

$T_{\lambda}^{F}(x)=\left\{z \in D: F(z, y)+\frac{1}{\lambda}\langle y-z, z-x\rangle \geq 0, \forall y \in D\right\}$.

If in the (4) we take $T=A_{F}$ a monotone bifunction, then we obtain the following Quasi Split Equilibrium Problem (QSEP):

Find

$x^{*} \in D ; F\left(x^{*}, x\right) \geq 0 \forall x^{*} \in D$ such that $A x^{*} \in C\left(x^{*}\right),(17$

and (5) is nothing but

$x_{k+1}=T_{\lambda_{k}}^{F}\left(x_{k}-\gamma A^{*}\left(I-P_{C\left(x_{k}\right)}\right) A x_{k}\right), k \in \mathbb{N}$.

It is well known that in this case, the strong monotonicity of $A_{F}$ is equivalent to

$$
F(x, y)+F(y, x) \leq \alpha\|x-y\|^{2} \text { for all } x, y \in D .
$$

\section{A special form of the implicit set.}

In many applications (see for example [2]) the set-valued mapping has the form $C(x)=K+\psi(x)$, where $K$ is a fixed closed subset in $H_{2}$ and $\psi: H_{1} \rightarrow H_{2}$ is a single-valued mapping. In this case, assumption on $C$ is satisfied provided the mapping $\psi$ is Lipschitz continuous. Indeed, it is not hard (using the relation below) to show that, if $\psi$ is $\kappa$-Lipschitz then assumption (6) satisfies with $\beta=2 \kappa$. Using the well known relation

$$
x=P_{K+v}(u) \Leftrightarrow x-v=P_{K}(u-v),
$$

Algorithm can be rewritten in the simpler form

$$
\begin{aligned}
& x_{k+1}=J_{\lambda_{k}}^{T}\left(x_{k}-\gamma_{k} A^{*}\left(A x_{k}-\psi\left(x_{k}\right)\right.\right. \\
& \left.+P_{K}\left(A x_{k}-\psi\left(x_{k}\right)\right)\right), k \in \mathbb{N} .
\end{aligned}
$$

\section{Conclusion}

Only the existence of solutions to a implicit split feasibility problem has been considered in [9]. Also, only some algorithms are mentioned! To the best of our knowledge, nothing has been done concerning the construction of solutions in this case. Inspired by this work and to overcome the difficulties that arise in applying the Banach principle, we proposed an implicit feasibility null-point problem and study the convergence of a related algorithm. Applications to some applied nonlinear analysis problems are also provided. The techniques used in solving our problem are strongly based on the resolvent mapping which depends on a parameter. The latter allows more flexibility and an appropriate choice amounts to assume mild assumptions on the data.

\section{References}

[1] H. H. Bauschke and J.M. Borwein, On projection algorithms for solving convex feasibility problems, SIAM Review, 38, 367-426 (1996).

[2] A. Bensoussan and J.Lions, Application des Inquations Variationelles en Control et en Stochastiques, Dunod, Paris, (1978).

[3] M. Bounkhel, L. Tadji and A. Hamdi, Iterative schemes for variational problems, J. Inequal. Pure and Appl. Math., Art. 14, 4, (2003).

[4] Ch. Byrne, Iterative oblique projection onto convex sets and the split feasibility problem. Inverse Problems, 18, 441-453 (2002).

[5] Ch. Byrne, Y. Censor, A. Gibali, S. Reich, Weak and Strong Convergence of Algorithms for the Split Common Null Point Problem, Journal of Nonlinear and Convex Analysis, 13, 759-775 (2012).

[6] Y. Censor and A. Segal, The split common fixed point problem for directed operators, Journal of Convex Analysis, 16, 587-600 (2009).

[7] N. Hadjisavvas and H. Khatibzadeh, Maximal monotonicity of bifunctions, Optimization, 59, 147-160 (2010).

[8] A. Moudafi, A note on Quasi Split Null-point Feasibility Problems, accepted for publication in Journal of Nonlinear Analysis and Optimization, (2013).

[9] M. A. Noor and K. I. Noor, Some new classes of quasi split feasibility problems, Applied Mathematics \& Information Sciences, 7, 1547-1552 (2013).

[10] R. T. Rockafellar, Monotone operators and the proximal point algorithm, SIAM J. Control and Optimization, 877-898 (1976).

[11] H.-K. Xu, Iterative methods for the split feasibility problem in infinite-dimensional Hilbert spaces, Inverse Problems, 26, 17 (2010). 


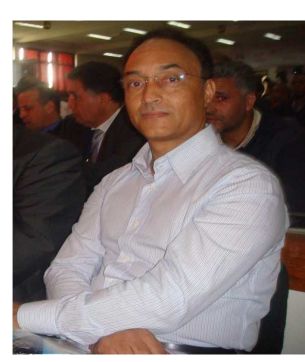

\begin{abstract}
Abdellatif Moudafi earned his $\mathrm{PhD}$ degree from Montpellier University, France (1991) in the field of Applied Mathematics (Nonlinear Analysis and Optimization). He has vast experience of teaching and research at university levels in various institutions including
\end{abstract} Montpellier, Clermont-Ferrand, Limoges, Marrakech, Guadeloupe and Martinique. His field covers many areas of Applied Mathematics such as Optimization, Variational Analysis, Asymptotic Analysis, Fixed-point problems and equilibria. He has obtained the Grant of Scientific Excellence for the periods 2001/2005, 2006/2010, and 2011/2015. He is currently member of the Editorial Board of several reputed international journals of Mathematics. He has more than 100 research papers to his credit which were published in leading world class journals

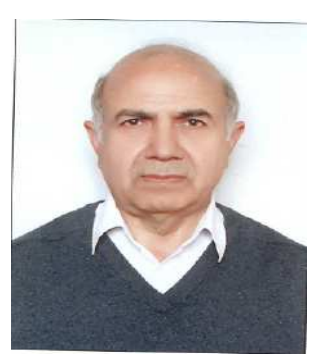

M. Aslam Noor earned his $\mathrm{PhD}$ degree from Brunel University, London, UK (1975) in the field of Applied Mathematics(Numerical Analysis and Optimization). $\mathrm{He}$ has vast experience of teaching and research at university levels in various countries including Pakistan, Iran, Canada, Saudi Arabia and United Arab Emirates. His field of interest and specialization is versatile in nature. It covers many areas of Mathematical and Engineering sciences such as Variational Inequalities, Operations Research and Numerical Analysis. He has been awarded by the President of Pakistan: President's Award for pride of performance on August 14, 2008, in recognition of his contributions in the field of Mathematical Sciences. He was awarded HEC Best Research paper award in 2009. He has supervised successfully several Ph.D and MS/M.Phil students. He is currently member of the Editorial Board of several reputed international journals of Mathematics and Engineering sciences. He has more than 750 research papers to his credit which were published in leading world class journals. 